\title{
Epilepsy in Children - Brain Growth and Behavior
}

\section{Hideaki Kanemura $^{1 *}$ and Masao Aihara ${ }^{2}$}

${ }^{1}$ Department of Pediatrics, Faculty of Medicine, University of Yamanashi, Japan

2Interdisciplinary Graduate School of Medicine and Engineering, University of Yamanashi, Japan

\begin{abstract}
The degree of behavioral problems associated with epilepsy in children is greater than would be expected on the basis of the existence of a chronic illness alone. Involvement of the frontal lobe such as atypical evolution of benign childhood epilepsy with centrotemporal spikes (BCECTS), epilepsy with continuous spike-waves during slow sleep (CSWS), and frontal lobe epilepsy (FLE) are characterized by impairment of neuropsychological abilities, and are frequently associated with behavioral disorders. In volumetric studies using three-dimensional (3D) magnetic resonance imaging (MRI), frontal and prefrontal lobe volumes revealed growth disturbance in patients with atypical evolution of BCECTS, CSWS, and FLE compared with those of normal subjects. These studies also revealed that in patients with shorter seizure durations and abnormal EEG periods, developmental abnormalities of the frontal lobe were soon restored to a more normal growth ratio. Conversely, growth disturbances of the prefrontal lobes were persistent in the patients with longer seizure durations and abnormal EEG periods. These findings suggest that seizure and the duration of paroxysmal anomalies may be associated with prefrontal lobe growth abnormalities, which are associated with neuropsychological problems. The urgent suppression of these seizure and EEG abnormalities may be necessary to prevent the progression of neuropsychological impairments.
\end{abstract}

Keywords: Benign childhood epilepsy with centrotemporal spikes (BCECTS); Epilepsy with continuous spikes and waves during slow sleep (CSWS); Frontal lobe epilepsy (FLE); Brain damage; Frontal lobe; Prefrontal lobe; Frontal lobe dysfunction; Brain volumetry

\section{Introduction}

Many children with epilepsy suffer from interictal disturbances in behavior that can contribute significantly to their illness and in some cases constitute the major disability. There remains some controversy on this subject, and it is generally accepted by most experts in the field that the degree of behavioral problems associated with epilepsy in children is greater than would be expected on the basis of the existence of a chronic illness alone. In the past, the emphasis has been on the outcome in terms of seizure control. However, with regard to other factors such as cognition and behavior, the value of pediatric epilepsy syndrome classification is increasingly recognized.

The frontal lobes of the brain constitute more than a third of the human cerebral cortex and are characterized by a complex functional organization supporting higher level integration circuits. The prefrontal parts are bidirectionally connected with all areas of association cortex in the brain, as well as with limbic structures and subcortical regions, including several of the thalamic nuclei and hypothalamus. This extensive system of connections makes it possible for the prefrontal cortex to receive information from practically all parts of the brain as well as to influence information processing in those parts. Prefrontal function can show an unusually long period of increased vulnerability, in which neurons and glial cells are readily affected by many factors including genetics, hormones, and external insults, such as infections, toxins, and trauma [1]. Among the cortical regions, the prefrontal cortex appears highly vulnerable to repeated seizures [2].

In general, focal epilepsies are often associated with neuropsychological, behavioral and emotional problems that can also affect a patient's adaptive functioning [3]. Accordingly, a frontal localization of the epileptic focus correlates with executive dysfunctions. Frontal lobe epilepsies, as with other focal epilepsies, were formerly considered to be the consequences of overt or obscure brain lesions. However, frontal lobe epilepsy (FLE) is poorly understood. Seizures of frontal lobe origin are difficult to control [4]. In addition, children with FLE manifest significant psychosocial problems relative to normative standards [4].

In contrast, several studies have reported a higher incidence of cognitive and behavioral difficulties associated with frontal lobe dysfunctions in children with other epilepsy syndromes such as benign childhood epilepsy with centrotemporal spikes (BCECTS) or epilepsy with continuous spike and waves during slow sleep (CSWS). These studies suggest that management of treatment may be required to remit seizures as early as possible to achieve optimal prognosis for the cognitive and behavioral outcome of children with several epilepsies.

The maturation process, which is defined as the process of becoming mature, of the frontal lobes is protracted. Thus, the discussion regarding the possible consequences of seizures in the immature brain has been long and contentious. However, authors of recent review articles have concluded that seizures impair the developing brain, which is defined as the processes that generate, shape, and reshape the nervous system [5]. The growth of hippocampus and neocortex cells and especially the increase in volume of the neuropil require particular mention because a paucity of dendrites and delayed synapse formation or a lack of adequate glial cells would reduce the total volume of the neural tissue, compared with normal age-matched controls [6]. The likelihood that even morphological damage may depend on the age at which epileptic seizures develop must be taken into account [7]. In children, therefore, repeated seizures may lead to cognitive and behavioral impairments.

*Corresponding author: Hideaki Kanemura, Department of Pediatrics, Faculty of Medicine, University of Yamanashi, Japan, Tel: +81-55-273-9606; Fax: +81-55273-6745; E-mail: ykimu@yamanashi.ac.jp

Received March 23, 2013; Accepted May 13, 2013; Published May 20, 2013

Citation: Kanemura H, Aihara M (2013) Epilepsy in Children - Brain Growth and Behavior. J Neurol Neurophysiol S2: 006 doi:10.4172/2155-9562.S2-006

Copyright: (c) 2013 Kanemura H, et al. This is an open-access article distributed under the terms of the Creative Commons Attribution License, which permits unrestricted use, distribution, and reproduction in any medium, provided the original author and source are credited. 


\section{Brain Volumetric Analyses in Central Nervous System Disease}

Magnetic resonance imaging (MRI)-based volumetry has become established as a versatile, reliable method for investigating the biology of the human brain [8]. Stronger correlations have been found between disability and the quantitative assessment of cerebral atrophy in various brain diseases using MRI. Examinations using three-dimensional (3D) MRI have confirmed that decreased frontal lobe volume correlates with clinical regression in subacute sclerosing panencephalitis [9]. These results suggest that volumetric analysis of the brain may predict function in the affected regions. Accordingly, brain volume measurement is a useful metric for characterizing abnormal development due to disease.

\section{Volumetric analysis of prefrontal cortex in children with epilepsy}

\section{Benign childhood epilepsy with centrotemporal spikes (BCECTS)}

BCECTS is idiopathic, age-specific, localization-related epilepsy with a benign course [10]. However, increasingly a considerable proportion of children with BCECTS have been shown to present behavioral problems such as hyperactivity and impulsivity [11]. Such impairments are more prevalent in children with atypical electroencephalogram (EEG) features or atypical seizure semiology $[12,13]$. Serial changes in the frontal and prefrontal cortex volumes using 3D-MRI in BCECTS with or without cognitive/behavioral problems were evaluated $[14,15]$. This study showed that frontal and prefrontal lobe volumes revealed growth disturbance in BCECTS with cognitive/behavioral problems compared with BCECTS without cognitive/behavioral problems and control subjects $[14,15]$. In addition, this study showed that the prefrontal-to-frontal lobe volume ratio increased serially in BCECTS without cognitive/behavioral problems, similar to controls, but was stagnant or decreased in BCECTS with cognitive/behavioral problems. Moreover, prefrontal growth also revealed more rapid recovery in BCECTS with cognitive/behavioral problems with a shorter active seizure period in that study (Figure 1A) [15]. These findings suggest that clinical symptoms, such as frequent seizures, and clinical investigations, such as paroxysms on EEG, might be associated with prefrontal lobe growth disturbance, which relates to neuropsychological problems in children with epilepsy.

\section{Epilepsy with continuous spike and waves during slow sleep (CSWS)}

Although the most prominent feature of epilepsy is seizure, the condition may also involve mental health problems, including hyperactivity, inattention, learning disabilities, other disease-related quality of life impairments, and psychopathology [16]. CSWS is a rare disease that affects children and is associated with deterioration of one or more cognitive functions, behavioral disturbances, spike and spike-wave discharges increased during slow wave sleep, and epileptic seizures. The majority of patients with CSWS present with seizures. Similarly, neuropsychological impairment occurs in almost all cases of CSWS, usually occurring with the detection of electrical status epilepticus during slow sleep (ESES) and representing one of the crucial signs of the syndrome [17]. CSWS is characterized by impaired neuropsychological abilities, frequently associated with behavioral disorders such as hyperactivity. These manifestations strongly correlate with frontal lobe dysfunction [18]. In previous studies, frontal and prefrontal lobe volumes of patients with CSWS were measured using
3D-MRI $[19,20]$. Though all patients had normal findings on routine MRI studies, they showed behavioral disturbances and/or cognitive impairments. The patients with a CSWS duration of less than 6 months showed remarkable improvements of behavioral disturbances. By contrast, patients with CSWS for longer than one year exhibited progressive cognitive and behavioral deterioration, even after seizure and paroxysmal EEG activities disappeared [20]. In these studies, frontal and prefrontal lobe volumes revealed growth disturbance in all CSWS patients compared with those of normal subjects [20]. In addition, prefrontal to frontal lobe volume ratios increased serially in normal subjects, whereas the ratios decreased in all CSWS patients. Moreover, these studies revealed that in the patients with shorter seizure durations and CSWS periods, prefrontal to frontal lobe volume ratios were soon restored to normal values, whereas growth disturbances of the prefrontal lobes were persistent in the patients with longer seizure durations and CSWS periods (Figure 1B) [20]. These findings suggest that seizure and the duration of paroxysmal abnormalities may be associated with prefrontal lobe growth disorders, which are associated with neuropsychological problems such as behavioral impairments.

\section{Frontal lobe epilepsy (FLE)}

It has been reported that seizure semiology of children is different from that of adults with FLE $[21,22]$. The discussion regarding the possible consequences of seizures in the immature brain has been long and contentious. The likelihood that even morphological damage may depend on the age at which epileptic seizures develop must be taken into account [23]. Repeated seizures may lead to cognitive and behavioral impairments. On the basis of these observations, prospective measurements of frontal and prefrontal lobe volumes using 3D-MRIbased volumetry were performed in patients with FLE presenting with a similar seizure history [2]. Frontal and prefrontal lobe volumes and the prefrontal-to-frontal lobe volume ratio increased serially in FLE patients without cognitive/behavioral impairments in a similar manner to controls. In contrast, frontal and prefrontal lobe volumes showed no obvious growth during the active seizure period in two FLE patients with cognitive/behavioral impairments. Prefrontal-to-frontal lobe volume ratio was stagnant or showed reduction during the active seizure period in the FLE patients with cognitive/behavioral impairments. The patient with a shorter active seizure period soon achieved a restored growth ratio. Conversely, growth ratio was delayed in patients with a longer active seizure period. Growth of the prefrontal lobe volume gradually normalized in all patients after seizure disappearance (Figure 1C) [2].

\section{Pathological Mechanisms Underlying Cognitive and Behavioral Impairments in Children with Epilepsy}

The effects of recurrent seizures are even more hotly debated. However, given the frequent occurrence of seizures in the children, and the unquestionable risk of vigorous medical treatment, the question of vulnerability of the developing brain to seizure-induced damage acquires great importance. Indeed, while the majority of children with epilepsy do well, a small percentage of children with epilepsy have cognitive and behavioral problems. A frontal localization of the epileptic focus correlates with executive dysfunction. Executive skills are also implicated in motor planning, controlling impulses and regulating behavior, as well as in emotional responses, behavioral and social actions [24]. They consist of those capacities that enable a person to engage successfully in independent, purposeful, and self-serving behavior [25].

MRI is currently the most effective method for detecting gross 
Citation: Kanemura H, Aihara M (2013) Epilepsy in Children - Brain Growth and Behavior. J Neurol Neurophysiol S2: 006 doi:10.4172/2155-9562. S2-006

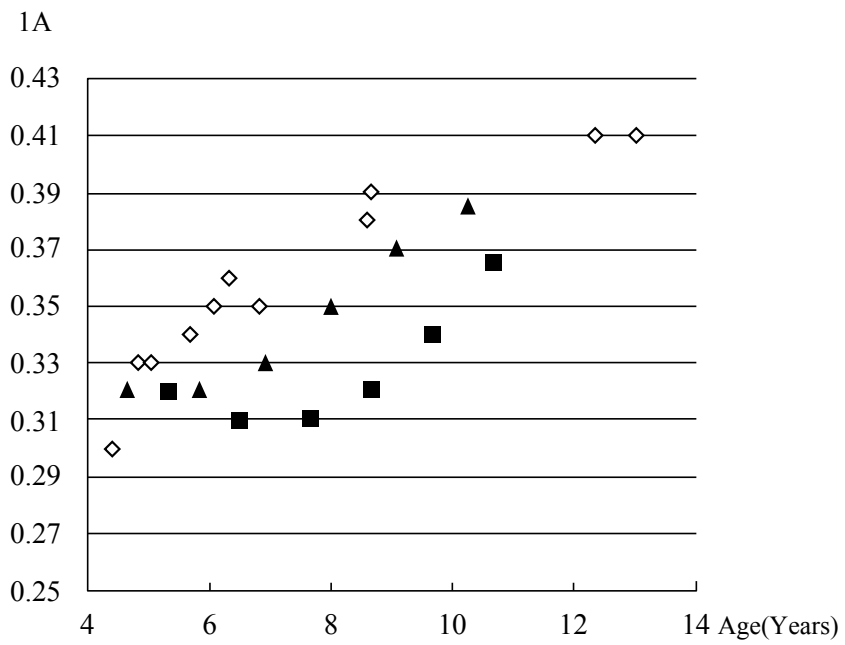

$1 \mathrm{~B}$

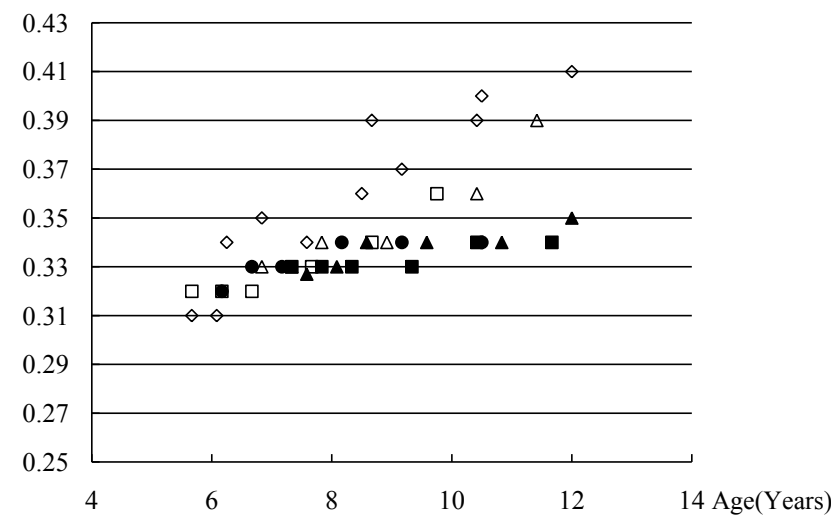

$1 \mathrm{C}$

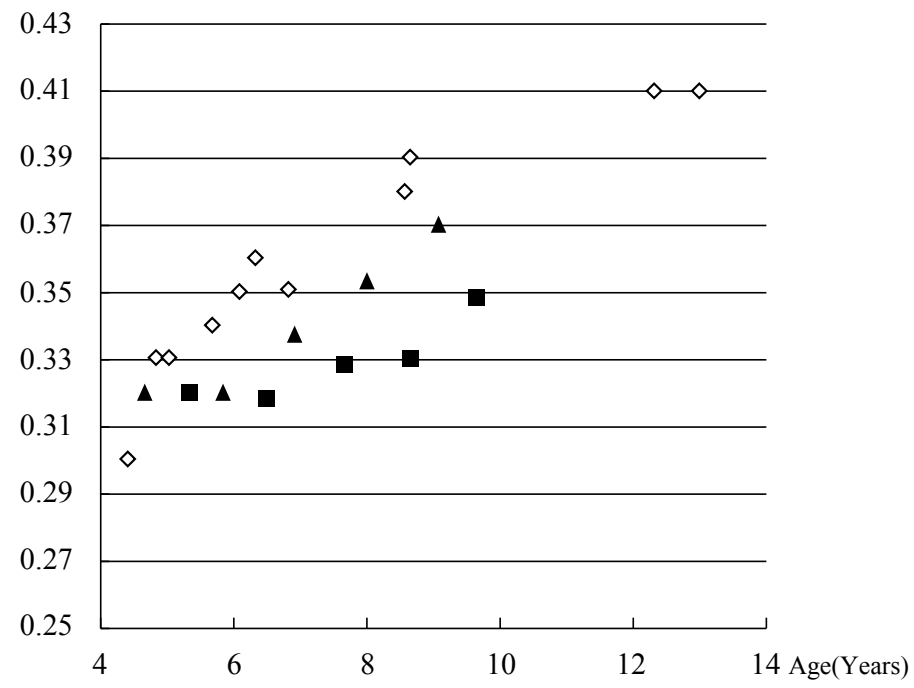

Figure 1: Serial changes in prefrontal-to-frontal lobe volume ratio in patients with BCECTS (A), CSWS (B), FLE (C) and control subjects. (A) scatter plots for BCECTS patients with cognitive/behavioral problems (longer seizure and abnormal EEG periods; closed squares, shorter seizure and abnormal EEG periods; closed triangles) and age-matched control subjects (open diamonds). (B) scatter plots for CSWS patients with longer seizure durations and CSWS periods (closed circles/squares/ triangles), with shorter seizure durations and CSWS periods (open squares/triangles), and age-matched controls (open diamonds). (C) scatter plots for FLE patients with cognitive/behavioral problems (longer seizure periods; closed squares, shorter seizure periods; closed triangles) and control subjects (open diamonds). BCECTS, benign childhood epilepsy with centrotemporal spikes; CSWS, epilepsy with continuous spikes and waves during slow sleep; FLE, frontal lobe epilepsy. 
structural lesions in patients with various brain diseases. Given the possibility that some functional changes may have structural correlates, MRI could also play a pivotal role in elucidating the mechanisms underlying epileptogenesis. Therefore, quantification of brain volume using 3D-MRI is a useful way of characterizing normal growth and abnormal development due to various diseases [26]. Furthermore, volumetric analysis of the brain may predict function in corresponding regions [14]. In BCECTS, prefrontal-to-frontal lobe volume ratio increased serially in patients without cognitive/behavioral problems similar to controls, but that was stagnant or decreased in patients with cognitive/behavioral problems. Moreover, prefrontal growth also revealed more rapid recovery in a patient with cognitive/behavioral problems with a shorter active seizure period in that study. Similarly in CSWS, seizure and the duration of paroxysmal anomalies may be associated with prefrontal lobe growth disorders, which are associated with neuropsychological problems and behavioral impairment. In FLE, frontal and prefrontal lobe volumes showed growth patterns similar to control subjects in patients without cognitive/behavioral impairments. In contrast, frontal and prefrontal lobe volumes and the prefrontal-tofrontal lobe volume ratio in particular showed growth disturbances during the seizure period. These findings suggest that repeated seizures combined with EEG abnormalities may lead to prefrontal growth disturbance, reflecting cognitive and behavioral impairments. Children with frontal lobe epilepsies such as BCECTS, CSWS, and FLE presenting with cognitive and behavior deficits may show frontal and prefrontal lobe retardation, which reflects poor frontal lobe functioning.

The developing brain appears to be more resistant to the toxic effects of glutamate than the mature brain [27]. As apoptosis plays an important role in early brain development, the immature brain may be particularly vulnerable to programmed cell death in response to seizures. These investigations have revealed strong evidence for the vulnerability of the immature brain to seizure-induced damage, which bears features of both necrotic and apoptotic death and contributes to synaptic reorganization [28]. In contrast, previous studies show that the prefrontal cortex is among the last cortical regions to reach full structural development with a long period of vulnerability $[1,26]$. Accordingly, prefrontal function and its disorders are not immediately apparent. These findings suggest that the prefrontal lobe may be highly vulnerable to repeated seizures among the cortical regions.

Carbamazepine sometimes exacerbates EEG abnormalities and behavioral/cognitive impairment. However, EEG abnormalities and behavioral/cognitive impairments in the patients with atypical evolution of BCECTS persisted even after discontinuation of CBZ. Therefore, it needs not be given consideration to effect of CBZ for the worse. In addition, valproate sodium (VPA) can induce reversible atrophy. However, the prefrontal lobe and the prefrontal-to-frontal lobe volume ratio in the present cases were restored to the growth ratio, although VPA was given through the clinical course. Therefore, it needs not be given consideration to effect of VPA for brain atrophy. However, it should be discussed the potential neurotoxic effects of antiepileptic medications that theoretically might interfere with growth of the immature brain. Further research will be needed to clarify this point.

\section{The Relationship between Cognitive/Behavioral Impairments and Seizure Recurrence}

There is limited and controversial data relating to the correlation between the frequency of seizures and neuropsychological findings. In early studies, frequent and severe seizures have been associated with behavioral problems [29,30]. Later, Schoenfeld et al. reported that the frequency of complex partial seizures in the previous year was the strongest predictor of behavioral problems [31]. Lendt et al. showed a reduction in behavioral problems in a group of children after successful surgery for epilepsy compared with children experiencing persistent focal seizures [32]. However, studies have shown no association between behavioral problems and more frequent seizures against these findings [33]. In more recent research by Austin et al., seizure recurrence significantly predicted behavioral problems [34]. According to their speculation, possible explanations for this relationship are summarized as follows: a) seizures and behavioral problems are associated because both are associated with similar underlying factors; b) seizure activity per se disrupts behavior; or c) children have a negative psychological response to seizures [34]. In brain volumetric studies with BCECTS or CSWS [9-11], active seizure period as frequent spike-waves coupled with the occurrence of frequent seizures may be associated with prefrontal lobe growth disturbance. In FLE using brain volumetry, damage to the frontal regions during childhood may interrupt normal maturational processes and organization, resulting in impairments to neurobehavioral development. Current research on frontal lobe epilepsies may clarify the factors that influence the volumetric abnormalities associated with seizure frequency. These results may support the proposal by Austin et al. that seizure recurrence may be a predictor of behavior issues. However, it is important first to consider that the majority of studies contain speculations on the qualitative analysis of the data without supporting statistical analyses [35]. Further research will be needed to address these limitations.

\section{Treatment of Children with Epilepsy Presenting with Cognitive/Behavioral Problems}

In considering outcomes for children with epilepsy, control of seizures must be weighed against the incidence of neurological impairments. Based on these volumetric and prospective studies, the most critical factor, from a therapeutic viewpoint, is whether seizures themselves can damage the brain. Management of treatment to remit seizures as soon as possible may be required to achieve optimal prognosis in epileptic children with cognitive or behavioral issues. Seizures originating from the frontal lobe may be difficult to control. A previous report found that seizure control was difficult, with only half of patients being successfully treated with anti-epileptic medications [4]. Although seizures and EEG abnormalities in atypical evolution of BCECTS and CSWS are age-dependent and self-limiting, cognitive and behavioral problems are often intractable before adolescence. The objective of the treatment is not necessarily a seizure-free state, but rather aims for improvements in alertness, cognitive function, mood and behavior.

Few anti-epileptic drugs (AEDs) may control atypical evolution of BCECTS, CSWS, and FLE, and none of them proved to be efficient in a randomized procedure. VPA alone or in combination with benzodiazepines (BDZ) is the treatment of choice for epilepsies with CSWS [17]. In addition, VPA in combination with BDZ and ethosuximide (ESM) was shown to decrease interictal epileptiform discharges in patients with CSWS and atypical evolution of BCECTS $[14,36]$. However, these suggestions are based primarily on case reports. In recent reports, the effect of levetiracetam (LEV) has been observed in CSWS [37-39], atypical evolution of BCECTS and FLE [39, 40]. Moreover, in patients with epilepsy presenting with secondarily bilateral synchrony (SBS) such as CSWS, atypical evolution of BCECTS and FLE, in whom cognitive and behavioral problems are serious, LEV showed effectiveness for seizure and EEG abnormalities [39]. 
Furthermore, LEV also decreased hyperactivity and impulsivity in these patients [39]. LEV may represent an important addition to the treatments available for refractory childhood epilepsies with SBS.

In conclusion, the durations of seizure (repeated seizures) combined with paroxysmal EEG abnormalities can induce brain damage and may be associated with pre-frontal region of the frontal lobes growth disturbances resulting in neuropsychological problems and ultimately neuropsychological outcomes in children with involvement of the frontal lobe such as atypical evolution of BCECTS, CSWS and FLE. To prevent these disturbances and problems in children with epilepsy, management of treatment may be required to remit seizures and EEG abnormalities as early as possible to achieve optimal prognosis for children with involvement of frontal lobe epilepsies. It is recommended that MRI performed in children with epilepsy should routinely include volumetric analysis of the cerebrum, if appropriate.

\section{References}

1. Dobbing J, Sands J (1971) Vulnerability of developing brain. IX. The effect of nutritional growth retardation on the timing of the brain growth-spurt. Biol Neonate 19: 363-378.

2. Kanemura H, Sano F, Tando T, Sugita K, Aihara M (2012) Repeated seizures induce prefrontal growth disturbance in frontal lobe epilepsy. Brain Dev 34: $175-180$

3. Cornaggia CM, Beghi M, Provenzi M, Beghi E (2006) Correlation between cognition and behavior in epilepsy. Epilepsia 47 Suppl 2: 34-39.

4. Sinclair DB, Wheatley M, Snyder T (2004) Frontal lobe epilepsy in childhood. Pediatr Neurol 30: 169-176.

5. Holmes GL, Ben-Ari Y (2001) The neurobiology and consequences of epilepsy in the developing brain. Pediatr Res 49: 320-325.

6. Sarnat HB, Flores-Sarnat L, Trevenen CL (2010) Synaptophysin immunoreactivity in the human hippocampus and neocortex from 6 to 41 weeks of gestation. J Neuropathol Exp Neurol 69: 234-245.

7. Mares $P(2008)$ Cognitive and affective effects of seizures: immature developing animals. In: Schachter SC, Holmes GL, Kasteleijn-Nolst Trenite DGA, editors. Behavioral aspects of epilepsy: Principles and practice. New York: Demos 2933.

8. Caviness VS Jr, Lange NT, Makris N, Herbert MR, Kennedy DN (1999) MRIbased brain volumetrics: emergence of a developmental brain science. Brain Dev 21: 289-295

9. Kanemura H, Aihara M, Okubo T, Nakazawa S (2005) Sequential 3-D MRI frontal volume changes in subacute sclerosing panencephalitis. Brain Dev 27: 148-151.

10. [No authors listed] (1989) Proposal for revised classification of epilepsies and epileptic syndromes. Commission on Classification and Terminology of the International League Against Epilepsy. Epilepsia 30: 389-399.

11. Chevalier H, Metz-Lutz MN, Segalowitz SJ (2000) Impulsivity and control of inhibition in Benign Focal Childhood Epilepsy (BFCE). Brain Cogn 43: 86-90.

12. Massa R, de Saint-Martin A, Carcangiu R, Rudolf G, Seegmuller C, et al. (2001) EEG criteria predictive of complicated evolution in idiopathic rolandic epilepsy. Neurology 57: 1071-1079.

13. Vinayan KP, Biji V, Thomas SV (2005) Educational problems with underlying neuropsychological impairment are common in children with Benign Epilepsy of Childhood with Centrotemporal Spikes (BECTS). Seizure 14: 207-212.

14. Kanemura $\mathrm{H}$, Aihara $\mathrm{M}$ (2009) Growth disturbance of frontal lobe in BCECTS presenting with frontal dysfunction. Brain Dev 31: 771-774.

15. Kanemura H, Hata S, Aoyagi K, Sugita K, Aihara M (2011) Serial changes of prefrontal lobe growth in the patients with benign childhood epilepsy with centrotemporal spikes presenting with cognitive impairments/behavioral problems. Brain Dev 33: 106-113.

16. Noeker M, Haverkamp-Krois A, Haverkamp F (2005) Development of mental health dysfunction in childhood epilepsy. Brain Dev 27: 5-16.

17. Tassinari CA, Rubboli G, Volpi L, Billard C, Bureau M (2005) Electrical status epilepticus during slow sleep (ESES or CSWS) including acquired epileptic aphasia (Landau-Kleffner syndrome). In: Epileptic syndromes in infancy, childhood and adolescence. (4thed), Roger J, Bureau M, Dravet C, Genton P, Tassinari CA, Wolf P, eds. John Libbey, London 295-314.

18. Fuster JM (1997) The Prefrontal Cortex: Anatomy, Physiology, and Neuropsychology of the Frontal Lobe (3rd ed.), Lippincott-Raven, Philadelphia.

19. Kanemura H, Sugita K, Aihara M (2009) Prefrontal lobe growth in a patient with continuous spike-waves during slow sleep. Neuropediatrics 40: 192-194.

20. Kanemura H, Aihara M (2011) Sequential prefrontal lobe volume changes in epileptic patients with continuous spikes and waves during slow sleep. In Gadze ZP (Ed). Epilepsy in Childen - Clinical and Social Aspects. Croatia: INTECH 13-24.

21. Fogarasi A, Janszky J, Faveret E, Pieper T, Tuxhorn I (2001) A detailed analysis of frontal lobe seizure semiology in children younger than 7 years. Epilepsia 42: 80-85.

22. Vigevano F, Fusco L (1993) Hypnic tonic postural seizures in healthy children provide evidence for a partial epileptic syndrome of frontal lobe origin. Epilepsia 34: $110-119$

23. Holmes GL, Ben-Ari Y (2001) The neurobiology and consequences of epilepsy in the developing brain. Pediatr Res 49: 320-325

24. Lezak MD, Howieson DB, Loring DW (2004) Neuropsychological assessment 4th ed. Oxford University Press; New York.

25. Gioia G, Isquith P, Guy S (2001) Assessment of executive functions in children with neurological impairment. In: Simeosson R, Rosenthal S, eds. Psychological and developmental assessment: children with disabilities and chronic conditions. The Guildford Press; New York 317-356.

26. Kanemura H, Aihara M, Aoki S, Araki T, Nakazawa S (2003) Development of the prefrontal lobe in infants and children: a three-dimensional magnetic resonance volumetric study. Brain Dev 25: 195-199.

27. Marks JD, Friedman JE, Haddad GG (1996) Vulnerability of CA1 neurons to glutamate is developmentally regulated. Brain Res Dev Brain Res 97: 194-206.

28. Sankar R, Shin D, Mazarati AM, Liu H, Katsumori H, et al. (2000) Epileptogenesis after status epilepticus reflects age- and model-dependent plasticity. Ann Neurol 48: 580-589.

29. Hoare $P$ (1984) The development of psychiatric disorder among schoolchildren with epilepsy. Dev Med Child Neurol 26: 3-13.

30. Austin JK, Risinger MW, Beckett LA (1992) Correlates of behavior problems in children with epilepsy. Epilepsia 33: 1115-1122.

31. Schoenfeld J, Seidenberg M, Woodard A, Hecox K, Inglese C, et al. (1999) Neuropsychological and behavioral status of children with complex partial seizures. Dev Med Child Neurol 41: 724-731.

32. Lendt M, Helmstaedter C, Kuczaty S, Schramm J, Elger CE (2000) Behavioura disorders in children with epilepsy: early improvement after surgery. J Neurol Neurosurg Psychiatry 69: 739-744.

33. Mitchell WG, Scheier LM, Baker SA (1994) Psychosocial, behavioral, and medical outcomes in children with epilepsy: a developmental risk factor model using longitudinal data. Pediatrics 94: 471-477.

34. Austin JK, Dunn DW, Caffrey HM, Perkins SM, Harezlak J, et al. (2002) Recurrent seizures and behavior problems in children with first recognized seizures: a prospective study. Epilepsia 43: 1564-1573

35. Vago C, Bulgheroni S, Franceschetti S, Riva D (2011) Frontal lobe epilepsies: neuropsychological and behavioral consequences in children. In: Gadze ZP (Ed). Epilepsy in Childen - Clinical and Social Aspects. Croatia: INTECH 187212.

36. Marescaux C, Hirsch E, Finck S, Maquet P, Schlumberger E, et al. (1990) Landau-Kleffner syndrome: a pharmacologic study of five cases. Epilepsia 31 768-777.

37. Aeby A, Poznanski N, Verheulpen D, Wetzburger C, Van Bogaert P (2005) Levetiracetam efficacy in epileptic syndromes with continuous spikes and waves during slow sleep: experience in 12 cases. Epilepsia 46: 1937-1942.

38. Chhun S, Troude P, Villeneuve N, Soufflet C, Napuri S, et al. (2011) A prospective open-labeled trial with levetiracetam in pediatric epilepsy syndromes: continuous spikes and waves during sleep is definitely a target Seizure 20: 320-325. 
Citation: Kanemura H, Aihara M (2013) Epilepsy in Children - Brain Growth and Behavior. J Neurol Neurophysiol S2: 006 doi:10.4172/2155-9562. S2-006

39. Kanemura H, Sano F, Sugita K, Aihara M (2013) Effects of levetiracetam on seizure frequency and neuropsychological impairments in children with refractory epilepsy with secondary bilateral synchrony. Seizure 22: 43-47.
40. Kanemura H, Sano F, Tando T, Sugita K, Aihara M (2013) Efficacy and safety of add-on levetiracetam in refractory childhood epilepsy. Brain Dev 35: 386391.

This article was originally published in a special issue, Epilepsy: Current

Trends handled by Editor(s). Dr. Espinosa PS, Centro Internacional en

Neurociencias, USA 